\title{
Estratégias de Coping de Pacientes Oncológicos em Tratamento Radioterápico
}

\author{
Wanderley de Paula Júnior \\ Daniela Sacramento Zanini ${ }^{1}$ \\ Pontifícia Universidade Católica de Goiás
}

\begin{abstract}
RESUMO - Este estudo analisa estresse, estratégias de coping, resiliência e bem-estar subjetivo em pacientes oncológicos em tratamento radioterápico. Sessenta pessoas, com idades entre 26 a 82 anos ( 20 homens e 40 mulheres) responderam as escalas de Bem-Estar Subjetivo, Inventário de Resiliência e de Sintomas de Estresse e Coping Response Inventory - Adult Form (CRI-A). Os resultados apontam que o uso da estratégia de coping direta esteve associado a nível mais elevado de resiliência e afeto positivo, enquanto o uso da estratégia de evitação pode aumentar a percepção de afeto negativo e diminuir o relato de afeto positivo. Conclui-se que as estratégias de coping podem interferir no bem-estar subjetivo e podem estabelecer relação significativa com fatores de resiliência em paciente oncológico em radioterapia.
\end{abstract}

Palavras-chave: câncer; radioterapia; coping.

\section{Coping Strategies of Cancer Patients Undergoing Radiotherapy Treatment}

\begin{abstract}
This study analyzes stress, coping strategies, resilience and subjective well-being of cancer patients undergoing radiotherapy. Sixty subjects, between 26 to 82 years old (20 man and 40 woman), answered the Subjective Well-Being Scale, the Resilience and the Stress Symptoms Inventory and the Coping Response Inventory - Adult Form (CRI-A). The results showed that the use of approach coping strategies is associated with a higher level of resilience and with positive affect, while using avoidant coping strategies can lead to an increased perception of negative affect and to a lower report of positive affect. The results of this study suggest that coping strategies are related to resilience factors and can interfere with the subjective well-being of cancer patients undergoing radiotherapy treatment.
\end{abstract}

Keywords: cancer; radiotherapy; coping.

Os estudos sobre resiliência iniciaram há mais de trinta anos. Nesta época, a resiliência era compreendida como as características inatas do indivíduo que lhe possibilitavam resistir e superar os estressores vivenciados (Grünspun, 2003).

Para Assis, Pesce e Avanci (2006) o conceito de resiliência é descrito pela psicologia e pela psiquiatria como a capacidade da pessoa de resistir às adversidades, considerando fatores intrínsecos e extrínsecos a ela. Assim, resiliência é a relativa resistência aos efeitos adversos provenientes de situações estressoras que proporcionam ao indivíduo um desenvolvimento satisfatório face às vivencias de adversidades significativas. O processo de resiliência, contudo, pode ser potencializado ou prejudicado quando, em face das adversidades significativas, o indivíduo sofre a influência dos fatores de proteção e/ou risco, respectivamente.

De acordo com Rutter (1987), os fatores de risco são aqueles que, estando presentes, aumentam a probabilidade do indivíduo de, na vivencia de adversidades significativas, ter seu desenvolvimento comprometido. $\mathrm{O}$ autor cita como exemplo algumas situações como a pobreza, as perdas afetivas, as enfermidades, o desemprego, as guerras, as calamidades entre outras.

Em contrapartida, os fatores de proteção são aqueles que, na presença de adversidades significativas, aumentam a probabilidade do indivíduo desenvolver-se bem (Jessor,

1 Endereço para correspondência: Departamento de Psicologia, Universidade Católica de Goiás, Avenida Universitária 1069, Setor Universitário, Caixa Postal 86, Goiânia, GO. CEP 74605-010, Telefone: 62 32155294, Fax: 62 32819853.E-mail: dazanini@yahoo.com
Van Den Bos, Vanderryn, Costa \& Turbin, 1995). Neste sentido, os fatores de proteção modificam, melhoram ou alteram a resposta a um resultado não adaptativo (Rutter, 1987). Contudo, Jessor et al. (1995) ressaltam que risco e proteção não são dois lados de uma mesma moeda. Ao contrário, a determinação dos fatores de risco e proteção deve ser processual e incluir uma análise situacional do problema enfrentado, seu impacto na saúde do indivíduo, seus recursos para fazer frente, as estratégias de enfrentamento utilizadas e o resultado obtido (Zanini \& Forns, 2005).

Em sua pesquisa Costa e Assis (2006) descrevem os seguintes fatores de proteção: fortalecimento de vínculos, relacionamentos emocionais positivos, autonomia (que implica em autodeterminação), auto-imagem positiva, comportamento pró-social e projeto de vida. Desta maneira, e segundo esses autores, pode-se fomentar possíveis fatores de proteção com o intuito de dar suporte para o enfrentamento e ressignificar as adversidades vividas. Mota, Benevides-Pereira, Gomes e Araújo (2006) acrescentam, ainda, como fatores de proteção as crenças religiosas ou religião, o favorecimento da comunicação e a busca de resolução de problemas.

Pesce et al. (2005) relatam correlação significativa entre resiliência e constructos como auto-estima, apoio social, satisfação na vida, saúde física, baixo nível de depressão, sensação de bem-estar e habilidade para lidar com problemas. Além disso, citando Masten e Garmezy (1985), apontam que os estudos sobre resiliência em crianças apresentam três variáveis como fatores de proteção: características de personalidade, como auto-estima, flexibilidade, habilidade 
para resolução de problemas; coesão e bom relacionamento com a família; e disponibilidade de suporte externo como a escola, grupo de pares, comunidade, que reforce as estratégias de coping para lidar com eventos estressores. Neste sentido, o coping, conforme postulado por Lazarus e Folkman (1984) como os esforços cognitivos e comportamentais para lidar com exigências internas ou externas à pessoa diante de situações de estresse e que são percebidos por esta como excedendo seus recursos, é de grande importância para a compreensão do processo de resiliência. Assim, pode-se dizer que "o conceito de coping permeia os estudos de fatores de risco e proteção e mecanismos de vulnerabilidade e resiliência" (Zanini \& Forns, 2005, p.72), pois para que haja coping, é necessária a vivência de uma situação potencialmente difícil ou estressora que coloque o bem-estar do indivíduo em risco de tal forma que, dependendo dos recursos disponíveis para este indivíduo ou fatores de proteção que este possua, apresentará um enfrentamento mais adaptativo e, portanto, resiliente, ou menos adaptativo e, consequentemente desenvolverá uma psicopatologia (Zanini \& Forns, 2005). Além disso, estudos atuais parecem demonstrar que determinadas estratégias de coping podem ser promotoras de resiliência (Coifman, Bonanno, Ray \& Gross, 2007, Lisboa et al., 2002).

Embora tradicionalmente os estudos em coping demonstrassem uma associação entre as estratégias de aproximação ao problema ou focadas no problema e melhor adaptação social ou saúde mental, por um lado, enquanto as estratégias de coping do tipo evitativas ou focadas na emoção estariam mais associadas à psicopatologia, por outro lado (Antoniazzi, Dell'Aglio \& Bandeira, 1998; Lazarus \& Folkman, 1984, Moos, 1993), a partir da década de 1990 as pesquisas em coping tenderam a enfatizar sua relação com saúde em detrimento da atenção dada até a década de 1980 à relação entre coping e psicopatologia (Aldwin \& Revenson, 1987; Zanini \& Forns, 2005). Tratou-se de focar os aspectos positivos do coping que possibilitassem o crescimento psicológico, a resiliência e a adaptação social (Herman-Stahl \& Pertersen, 1996, 1999; Herman-Stahl, Stemmler \& Pertersen, 1995; Holahan, Moos \& Schaefer, 1996). Como exemplo podemos citar o estudo de Heim, Valach e Schaffner (1997), ao analisar 74 pacientes com câncer de mama, observadas em período de três a cinco anos de intervalo. Os autores observaram que o relacionamento interpessoal positivo e as estratégias de enfrentamento de aproximação ou direcionadas para o problema auxiliavam na adaptação psicossocial por meio do suporte social e do auto-controle, enquanto que o coping de evitação (negação e fuga) interferia negativamente nessa adaptação.

Em um estudo semelhante em população brasileira, Gimenes (1997) pesquisou 120 mulheres submetidas à cirurgia de mastectomia e apontou que o bem-estar psicológico foi predito pelas seguintes variáveis: foco no aspecto positivo, coesão familiar, autoculpa, redução de tensão e complicações de saúde. Os resultados demonstraram que mulheres com níveis altos de bem-estar psicológico tinham famílias coesas e focavam nos aspectos positivos frente à mastectomia. Por outro lado, complicações de saúde e o uso de estratégias de coping como a autoculpa e tentativa de redução de tensão para lidar com a doença estiveram associados a níveis baixos de bem-estar psicológico.
Pesquisas com populações diferentes apresentam resultados semelhantes aos relatados acima. Por exemplo, Zanini, Forns e Kirchner (2005), em um estudo com a população espanhola, constataram que estratégias de coping de aproximação ou focadas no problema estavam positivamente relacionadas com o estado de saúde e o bem-estar, em oposição às estratégias evitativas ou focadas na emoção relacionadas a condutas psicopatológicas ou ao aumento de relatos de mal-estar psicológico em adolescentes.

A partir do exposto anteriormente o presente artigo objetiva investigar as diferenças entre homens e mulheres submetidos à radioterapia como consequência de tratamento oncológico no uso de estratégias de enfrentamento a problemas e no auto relato de sintomas de estresse, de características de resiliência e de bem estar subjetivo. Além disso, o presente estudo pretende, ainda, avaliar a influência do tipo de enfrentamento utilizado por estes pacientes sobre o auto-relato de sintomas de estresse, de bem-estar subjetivo e de resiliência nestes pacientes.

\section{Método}

\section{Participantes}

Participaram deste estudo 60 pacientes selecionados aleatoriamente entre aqueles que atendiam o serviço de radioterapia de um Hospital de referencia da região Centro-Oeste do Brasil no período de fevereiro a outubro de 2008 e que estavam entre a quarta e a trigésima quinta aplicação. A seleção da amostra se deu independente do tempo de diagnóstico, tipo de atendimento (se ambulatorial ou internação) ou tipo de enfermidade oncológica diagnosticada.

Dos 60 participantes desta pesquisa $40(66,70 \%)$ eram do sexo feminino e $20(33,3 \%)$ do sexo masculino. As idades variaram entre 26 a 82 anos de idade $(M=52 ; D P=13,25)$. A maioria possuía ensino fundamental incompleto $(51,7 \%)$, eram casados $(60 \%)$, possuíam filhos $(96,6 \%)$, moravam com a família $(88,3 \%)$ e eram católicos $(58,3 \%)$. Além disso, de acordo com diagnóstico no prontuário, $31,7 \%$ dos pacientes apresentaram câncer de mama, 16,7\% câncer de próstata, $23,3 \%$ câncer de útero, $11,7 \%$ câncer de cabeça e pescoço, $5 \%$ câncer de cérebro, câncer de pele e intestino 3,3\% cada e câncer de pulmão, reto e esôfago $1,7 \%$ cada um.

\section{Instrumentos}

Para este estudo foram utilizados o Coping Response Inventory - Adult Form (CRI - A) de Moos (1993), o Inventário de Resiliência (Mota, Benevides-Pereira, Gomes \& Araújo, 2006), a Escala Bem-Estar Subjetivo, de Albuquerque e Tróccoli (2004), o Inventário de Sintomas de Estresse, de Benevides-Pereira e Moreno-Jiménez (2002).

O CRI - A de Moos (1993) avalia oito tipos estratégias de coping diretas que posteriormente se agrupam em dois tipos de estratégias globais: de aproximação do problema e de evitação do problema. O instrumento divide-se em três partes. Na primeira, o participante da pesquisa des- 
creve uma situação estressante que vivenciou nos últimos doze meses, no caso da presente pesquisa pediu-se aos participantes que relatassem o evento mais estressante que vivenciaram em relação à realização de radioterapia. $\mathrm{Na}$ segunda parte, o participante avalia 10 perguntas sobre os seguintes temas: experiência prévia com o problema, prejuízos e responsabilidade no acontecimento do evento estressante. Na terceira parte, o participante responde a 48 perguntas sobre comportamentos que utiliza para enfrentar o problema. Essas perguntas são respondidas de acordo com quatro alternativas do tipo Likert, e geram as pontuações das oito estratégias de coping diretas. A pontuação das escalas globais se dá por meio do somatório das escalas diretas correspondentes ao foco de coping. Assim, a escala global de Aproximação do problema é formada pelas estratégias de Análise Lógica, Reavaliação Positiva, Busca de Apoio e Resolução de Problemas; enquanto a escala global de Evitação do Problema é formada pelo conjunto de estratégias Evitação Cognitiva, Aceitação Resignação, Busca de Gratificação Alternativa e Descarga Emocional (Moos, 1993). O instrumento apresenta qualidade psicométrica satisfatória com alphas entre 0,58 e 0,72 , condizente com as demais escalas de coping existentes (Moos, 1993). $\mathrm{Na}$ versão brasileira, ainda em fase de validação, estudos preliminares com a população de estudantes universitários e trabalhadores em geral, além de grupos de pacientes crônicos e com transtornos psiquiátricos leves, apontaram uma consistência interna entre baixa $(0,55$ e 0,59 para as oito escalas diretas) e boa $(0,80$ e 0,77 para as escalas globais de aproximação e evitação, respectivamente).

O Inventário de Resiliência compõe-se de quarenta itens que expressam ações ou atitudes afirmativas e os participantes assinalam numa escala de um a cinco, se estão totalmente em desacordo (1), em desacordo (2), nem de acordo, nem em desacordo (3), de acordo (4), e totalmente de acordo (5). Estes itens foram agrupados em seis fatores: inovação e tenacidade, hiperemotividade, assertividade, empatia, satisfação no trabalho e competência emocional. Este instrumento apresentou boa qualidade psicométrica com alfas de Cronbach para as escalas de 0,78 para inovação e tenacidade, 0,78 para hiperemotividade, 0,67 para assertividade, 0,64 para empatia, 0,65 para satisfação no trabalho, 0,66 para competência emocional (Benevides-Pereira \& Moreno-Jiménez, 2002; Mota et al., 2006).

A Escala de Bem-Estar Subjetivo de Albuquerque e Tróccoli (2004) está dividida em duas partes. A primeira parte da escala é composta de 47 itens que descrevem afetos positivos e negativos, devendo o sujeito responder como tem se sentido ultimamente numa escala tipo Likert de cinco pontos. Na segunda parte da escala, os 15 itens restantes, descrevem julgamentos relativos à avaliação de satisfação ou insatisfação com a vida, também respondidos numa escala tipo Likert de cinco pontos. O conjunto de itens são, posteriormente, agrupados em três fatores: afeto positivo (alfa de Cronbach $=0,95$ ); afeto negativo (alfa de Cronbach $=0,95$ ); satisfação com a vida versus insatisfação com a vida (alfa = 0,90) (Albuquerque \& Tróccoli, 2004).

O Inventário de Sintomas de Estresse (ISE), de Benevides-Pereira e Moreno-Jiménez (2002) é constituído de trinta itens afirmativos, com respostas dadas em escala do tipo Li- kert (0 para "nunca", 1 "raras vezes", 2 "moderadamente", 3 "frequentemente", 4 "assiduamente"), relativos aos sintomas referenciados na literatura como frequentes ou característicos de estresse no dia-a-dia. Estes itens agrupam-se formando os fatores: sintomas físicos (SF) e sintomas psicológicos (SP). A consistência interna das dimensões apresentou alfa de Cronbach de 0,72 para SF e 0,85 para SP (Benevides-Pereira $\&$ Moreno-Jiménez, 2002).

\section{Procedimento}

Após o projeto de pesquisa ter sido aprovado pelo Comitê de Ética em pesquisa os participantes foram abordados pelo pesquisador responsável ou auxiliar, orientados sobre os objetivos do estudo e convidados a participar do mesmo. Após aceitação, foram convidados a assinar o Termo de Consentimento Livre e Esclarecido.

Os participantes foram contactados na sala de radioterapia. As aplicações foram individuais e ocorreram em um único encontro. A ordem de aplicação dos instrumentos foi à seguinte: Coping Response Inventory - Adult Form (CRI - A); Inventário de Resiliência; Escala Bem-Estar Subjetivo; Inventário de Sintomas de Estresse. O tempo de aplicação dos instrumentos variou segundo condições médicas dos participantes. Em geral, o tempo médio de aplicação foi de duas horas.

\section{Análise de Dados}

A análise de dados desta pesquisa foi realizada por meio do pacote estatístico para Windows SPSS versão 14.0. Para avaliar o uso diferencial de estratégias de coping, de resiliência, de bem-estar subjetivo e de sintomas de estresse quanto ao sexo, utilizou-se a técnica de Kruskal-Wallis, devido ao número reduzido da amostra $(\mathrm{N}=60)$. Para avaliar a relação entre as variáveis analisadas procedeu-se um estudo correlacional de Pearson.

\section{Resultados}

Conforme dados da Tabela 1, as mulheres relataram um uso superior das estratégias de análise lógica $(Z=-2,36$ e p $=0,02)$, resolução de problemas $(Z=-3,08$ e $p=0,02)$ e descarga emocional $(Z=-2,96$ e $\mathrm{p}=0,00)$.

No Inventário de Resiliência observa-se que os fatores da escala que apresentaram resultados significativos foram hiperemotividade $(Z=-3,49$ e $p=0,00)$ e empatia $(Z=-2,30$ e $\mathrm{p}=0,02)$, enquanto na Escala de Bem-Estar Subjetivo o afeto negativo $(Z=-2,24$ e $p=0,02)$, satisfação com a vida $(Z=-2,21$ e $p=0,03)$ e insatisfação com a vida $(Z=-2,24$ e $p=0,02)$ foram significativos, sendo as mulheres as que referem mais afeto negativo e insatisfação com a vida enquanto os homens referem mais satisfação com a vida.

Em relação ao Inventário de Sintomas do Estresse, o estudo de comparação de médias segundo o sexo (Tabela 1) revelou que são as mulheres que relatam mais sintomas psicológicos em comparação aos homens $(Z=-2,98$ e $\mathrm{p}=0,00)$. 
Tabela 1. Média, desvio padrão e diferenças de médias segundo sexo, segundo a técnica de Kruskal Wallis, para as estratégias de coping, resiliência, bemestar subjetivo e sintomas de estresse.

\begin{tabular}{|c|c|c|c|c|}
\hline \multirow{3}{*}{ Variável } & \multicolumn{4}{|c|}{ Sexo } \\
\hline & Masculino & Feminino & \multirow{2}{*}{$\mathbf{Z}$} & \multirow{2}{*}{$p<$} \\
\hline & M (DP) & M (DP) & & \\
\hline \multicolumn{5}{|l|}{ Estratégias de coping } \\
\hline Análise lógica & $7,10(3,42)$ & $9,35(3,63)$ & $-2,36$ & 0,02 \\
\hline Resolução de problemas & $7,30(2,96)$ & $10,05(3,22)$ & $-3,08$ & 0,02 \\
\hline Descarga emocional & $3,05(2,05)$ & $5,25(2,74)$ & $-2,96$ & 0,00 \\
\hline Cri-Afrontamento & $33,85(10,46)$ & $41,10(8,35)$ & $-2,56$ & 0,01 \\
\hline \multicolumn{5}{|l|}{ Fatores de Resiliência } \\
\hline Hiperemotividade & $9,60(2,19)$ & $11,70(1,71)$ & $-3,49$ & 0,00 \\
\hline Empatia & $9,85(2,03)$ & $11,15(1,44)$ & $-2,30$ & 0,02 \\
\hline \multicolumn{5}{|c|}{ Fatores de Bem-Estar Subjetivo } \\
\hline Afeto negativo & $43,05(16,07)$ & $56,48(21,52)$ & $-2,24$ & 0,02 \\
\hline Satisfação com a vida & $31,85(4,23)$ & $28,25(6,40)$ & $-2,21$ & 0,03 \\
\hline Insatisfação com a vida & $17,60(4,17)$ & $20,35(4,81)$ & $-2,24$ & 0,02 \\
\hline \multicolumn{5}{|c|}{ Fator de Sintomas de Estresse } \\
\hline Sintomas Psicológicos & $10,11(8,41)$ & $18,97(11,22)$ & $-2,98$ & 0,00 \\
\hline
\end{tabular}

Tabela 2. Análise de correlação de Pearson entre coping, resiliência, bem-estar subjetivo e sintomas de estresse (N=60).

\begin{tabular}{|c|c|c|c|c|c|c|c|c|c|c|c|c|}
\hline \multirow[t]{2}{*}{ Coping } & \multicolumn{5}{|c|}{ Resiliência } & \multicolumn{3}{|c|}{ Bem-Estar Subjetivo } & \multicolumn{4}{|c|}{ Sintomas de estresse } \\
\hline & IT & $\mathrm{He}$ & A & $\mathrm{E}$ & ST & $\mathrm{CE}$ & $\mathrm{AP}$ & NA & SCV & $\mathrm{ICV}$ & $\mathrm{SF}$ & $\mathrm{SP}$ \\
\hline $\mathrm{AL}$ & & & & & & & & $0,390 * *$ & & $0,348 * *$ & & $0,449 * *$ \\
\hline $\mathrm{RP}$ & & & & & $0,311 *$ & & $0,467 * *$ & & $0,291 *$ & & & \\
\hline $\mathrm{BA}$ & & & & & $0,381 * *$ & & $0,350 * *$ & & & & & \\
\hline PS & & & & & & & $0,492 * *$ & & & & & \\
\hline $\mathrm{EC}$ & & & & & & & & $0,329 *$ & & & & $0,281^{*}$ \\
\hline AR & & & & & & & $-0,307^{*}$ & $0,271^{*}$ & $0,272 *$ & $0,421 * *$ & & $0,367^{*}$ \\
\hline BGA & & & & & & & $0,371 * *$ & & & & $-0,2$ & \\
\hline $\mathrm{DE}$ & & 0,3 & & & & & & $0,404 * *$ & $-0,261 *$ & $0,407 * *$ & & $0,387 * *$ \\
\hline
\end{tabular}

Apenas os valores significativos são apresentados na tabela acima.

*Correlação significativa a 0,05 (2-tailed). **Correlação significativa a 0,01 (2-tailed).

$\mathrm{AL}=$ Análise lógica; $\mathrm{RP}=$ Reavaliação positiva; $\mathrm{BA}=\mathrm{Busca}$ de apoio; $\mathrm{RS}=$ Resolução de problemas; $\mathrm{EC}=$ Evitação Cognitiva; $\mathrm{AR}=\mathrm{Aceitação} \mathrm{Resignação;}$ $\mathrm{BGA}=$ Busca de gratificação alternativa; $\mathrm{DE}=$ Descarga emocional. $\mathrm{IT}=$ Inovação e Tenacidade; He = Hiperemotividade; $\mathrm{A}=\mathrm{Assertividade} ; \mathrm{E}=$ Empatia; $\mathrm{ST}=$ Satisfação no Trabalho; $\mathrm{CE}=$ Competência Emocional. AP = Afeto Positivo; $\mathrm{AN}=$ Afeto Negativo; $\mathrm{SCV}=$ Satisfação com a Vida; ICV = Insatisfação com a Vida. $\mathrm{SF}=$ Sintomas Físicos; $\mathrm{SP}=$ Sintomas Psicológicos.

De acordo com a análise de correlação, pode-se observar que quanto mais o participante da pesquisa utilizava a estratégia de coping análise lógica, mais afeto negativo $(\mathrm{r}=0,390$; $\mathrm{p}=0,002)$, insatisfação com a vida $(\mathrm{r}=0,348 ; \mathrm{p}=0,006)$ foram relatados, assim como mais sintomas psicológicos $(\mathrm{r}=0,449$; $\mathrm{p}=0,001)$. Portanto, o uso de estratégias de enfrentamento do tipo análise lógica parece desencadear maior desconforto quanto ao bem-estar subjetivo e maior sintomas de estresse com características psicológicas, na amostra estudada.

Pode-se notar que a reavaliação positiva se relacionou com satisfação no trabalho $(\mathrm{r}=0,311 ; \mathrm{p}=0,016)$, com afeto positivo $(\mathrm{r}=0,467 ; \mathrm{p}=0,000)$ e com satisfação com a vida $(\mathrm{r}=0,291 ; \mathrm{p}=0,024)$.
Além disso, observa-se que quanto mais os participantes relataram ter utilizado de estratégia do tipo busca de apoio, mais relataram satisfação no trabalho $(\mathrm{r}=0,381 ; \mathrm{p}=0,003)$ e afeto positivo $(\mathrm{r}=0,350 ; \mathrm{p}=0,007)$. A estratégia de coping resolução de problema se correlacionou com afeto positivo $(\mathrm{r}=0,492 ; \mathrm{p}<0,000)$; enquanto evitação cognitiva se correlacionou com afeto negativo $(\mathrm{r}=0,329 ; \mathrm{p}=0,010)$ e com sintomas psicológicos $(r=0,281 ; p=0,036)$.

Quanto mais os sujeitos utilizaram estratégia de aceitação/ resignação, menos se observa o afeto positivo $(\mathrm{r}=-0,307$; $\mathrm{p}=0,019)$ e menos satisfação com a vida $(\mathrm{r}=-0,272 ; \mathrm{p}=0,039)$, e maior $\mathrm{o}$ auto relato de afeto negativo $(\mathrm{r}=0,271 ; \mathrm{p}=0,040)$, 
insatisfação com a vida $(r=0,421 ; \mathrm{p}=0,001)$ e sintomas psicológicos $(\mathrm{r}=0,367, \mathrm{p}=0,006)$.

A estratégia de coping busca de gratificação alternativa se relacionou positivamente com afeto positivo $(\mathrm{r}=0,371$; $\mathrm{p}=0,003)$ e inversamente com sintomas físicos $(\mathrm{r}=-0,266$; $\mathrm{p}=0,043)$.

A estratégia de coping descarga emocional apresentou relação significativa direta com os fatores hiperemotividade $(r=0,327, p=0,011)$, afeto negativo $(r=0,404 ; p=0,01)$, insatisfação com a vida $(r=0,407 ; p=0,001)$ e sintomas psicológicos $(\mathrm{r}=0,387 ; \mathrm{p}=0,003)$, e relação inversa com o fator satisfação com a vida $(r=-0,261 ; p=0,044)$. Aceitação/resignação e descarga emocional também são estratégias de enfrentamento que podem resultar em afetos negativos e menor satisfação com a vida, resultando em aparecimento de sintomas de estresse psicológicos.

Inovação e tenacidade, empatia e competência emocional são fatores de resiliência que não se correlacionaram, neste estudo, com os fatores de coping.

\section{Discussão}

Em relação ao primeiro objetivo deste estudo, investigar as diferenças entre homens e mulheres submetidos à radioterapia como consequência de tratamento oncológico no uso de estratégias de enfrentamento a problemas e no auto-relato de sintomas de estresse, de características de resiliência e de bem-estar subjetivo, os dados demonstraram diferenças significativas entre as médias de coping de homens e mulheres compatíveis com aquelas descritas por Moos (1993). Estes dados, além de serem concordantes com os apresentados por este autor, denota que as mulheres apresentam médias maiores do que os homens nas estratégias de análise lógica, resolução de problemas e descarga emocional.

Quanto ao instrumento de sintomas de estresse, foram as mulheres que apresentaram significativamente mais sintomas psicológicos quando comparadas com os homens. Bergelt, Lehmann, Beierlein e Koch, (2008) e Sehgal et al. (2008) apresentam resultados similares em suas pesquisas quanto ao predomínio de sintomas psicológicos de estresse em mulheres. Nestes estudos, as pacientes com câncer de mama reportaram maior efeito colateral, fadiga e problemas de sono do que os pacientes com câncer de próstata. Entretanto, durante o tratamento de radiação, os dois grupos apontaram efeitos negativos do estresse e dor. Porém, as pacientes com câncer de mama parecem recuperar mais lentamente quando consideramos o funcionamento geral e emocional relativos à qualidade de vida, segundo Bergelt et al. (2008). Em nosso estudo, os dados também indicaram o aparecimento mais frequente de sintomas psicológicos de estresse nas mulheres pesquisadas, porém percebe-se que as mesmas também buscaram mais estratégias de coping para superar os problemas, principalmente as estratégias de aproximação.

Heim et al. (1997) relatam que o relacionamento interpessoal positivo e as formas de enfrentamento de aproximação, voltadas para o problema, auxiliam na adaptação psicossocial através do suporte social e autocontrole, porém o coping de evitação (negação e fuga) interfere negativamente nesta adaptação. Este estudo assemelha-se à pesquisa de Gimenes
(1997) quanto à importância da combinação de fatores internos e externos para promoção do bem-estar, que apontou também para o uso de estratégias de aproximação, ou voltadas para o problema, como melhor forma de adaptação.

Algumas pesquisas descrevem que estratégias de coping de aproximação ou focadas no problema estão positivamente relacionados com o estado de saúde e o bem-estar. Em oposição, as estratégias evitativas ou focadas na emoção dificultam o desenvolvimento saudável e podem desencadear condutas psicopatológicas ou aumento de relatos de mal-estar psicológico (Lisboa et al., 2002; Zanini et al., 2005).

Assim, podemos dizer que o uso de estratégias de evitação do problema leva não só a um maior relato de afetos negativos como a uma menor percepção de afetos positivos, enquanto os pacientes que enfrentam seus problemas por meio de estratégias de enfrentamento direto relatam mais afetos positivos.

Em relação ao estudo correlacional, observa-se que a aceitação/resignação e descarga emocional também são estratégias de enfrentamento que estão associadas com afetos negativos e menor satisfação com a vida, resultando em aparecimento de sintomas de estresse psicológicos. Já as formas de enfrentamento através da busca de gratificação alternativa associam-se com um maior auto-relato de afeto positivo e menor probabilidade de surgimento de sintomas físicos para sintomas de estresse.

Inovação e tenacidade, como fatores de resiliência, embora não relacionem com a forma de enfrentamento do problema, denotam maior assertividade e satisfação no trabalho e consequentemente maior bem-estar subjetivo através do afeto positivo e menor aparecimento de sintomas psicológicos. Deste modo, podemos postular que para o paciente com câncer, em tratamento radioterápico, parece que as estratégias de coping que se aproximam dos problemas estão associadas a índices mais elevados de fatores característicos de resiliência como inovação e tenacidade, assim como relacionados a contextos de trabalho.

Competência emocional, um dos fatores da resiliência, não se correlacionou com nenhum dos fatores de coping, bem-estar subjetivo, sintomatologia de estresse e tampouco com os outros fatores da resiliência. É importante ressaltar que o fator competência emocional do instrumento de resiliência é composto somente por dois itens. Além disso, os dois itens referem-se à expressão verbal dos sentimentos. O baixo número de itens e a direção da competência emocional como capacidade de expressão verbal dos sentimentos podem interferir na qualidade da resposta. Estudos posteriores deveriam ser desenvolvidos a fim de verificar novas possibilidades de avaliação de competência emocional que não somente aquela associada à expressão verbal de sentimentos.

$\mathrm{Na}$ escala de bem-estar subjetivo, os participantes da pesquisa referiram utilizar mais afeto positivo e, portanto, obter mais satisfação com a vida do que afeto negativo e, consequentemente, menos insatisfação com a vida. Estudos apontam que os altos níveis de bem-estar psicológico, entre outros fatores, estavam relacionados com o foco nos aspectos positivos para lidar com os eventos (Gimenes,1997; Schroevers, Kraaij \& Garnefski, 2008).

Para Albuquerque e Tróccoli (2004), o bem-estar subjetivo tem três características fundamentais: a subjetividade, as 
medidas positivas e a avaliação global. Entretanto, quando fazemos referência aos estudos com pacientes oncológicos em radioterapia pode-se compreender que apesar do diagnóstico de câncer, dos efeitos colaterais e adversidades do tratamento, entre outros, é possível afirmar que resiliência não implica simplesmente na cura do câncer, mas a forma como a pessoa lida com a doença e tratamento em diferentes momentos deste processo. Resiliência, neste contexto, é a possibilidade de superar e ressignificar as situações adversas ao longo da própria vida de forma positiva.

Por fim, o presente artigo contribui para a elucidação da importância das estratégias de coping como ferramentas de trabalho em intervenções clínicas a pacientes oncológicos em radioterapia, na medida em que demonstra que o uso de determinadas estratégias de coping (sobretudo aquelas relacionadas a aproximação dos problemas) está associado a melhor adaptação. Do mesmo modo, o presente artigo reforça a ideia da desadaptação ou associação de mal-estar psicológico relacionado à estratégias do tipo evitação do problema. Contudo, essas informações devem ser tomadas com precaução devido ao número de participantes desta pesquisa ser pequeno, considerando a população brasileira com diagnóstico de câncer em tratamento radioterápico.

É necessário que sejam realizados mais estudos científicos sobre coping, resiliência, estresse e bem-estar subjetivo, com intuito de verificar as possíveis relações existentes entre essas categorias como decorrente de formas mais adaptativas para enfrentamento das adversidades resultando em melhor adaptação para estes pacientes.

\section{Referências}

Aldwin, C., \& Revenson, T. (1987). Does coping help? A reexamination of the relation between coping and mental health. Journal of Personality and Social Psychology, 53, 337-348.

Albuquerque, A.S., \& Tróccoli, B.T. (2004). Desenvolvimento de uma escala de bem-estar subjetivo. Psicologia: Teoria e Pesquisa, 20, 153-164.

Antoniazzi, A.S., Dell'Aglio, D.D., \& Bandeira, D.R. (1998). O conceito de coping: uma revisão teórica. Estudos de Psicologia, 3(2), 273-29.

Assis, S., Pesce, R., \& Avanci, J. (2006). Resiliência: Enfatizando a proteção dos adolescentes. Porto Alegre: Artmed.

Benevides-Pereira, A. M. T., \& Moreno-Jiménez, B. (2002). O Burnout em um grupo de psicólogos brasileiros. In A.M.T. Benevides-Pereira, (Ed.), Burnout: Quando o Trabalho Ameaça o Bem-Estar do Trabalhador (pp. 157-185). São Paulo: Casa do Psicólogo.

Bergelt, C., Lehmann, C., Beierlein, V., \& Koch, U. (2008). Quality of life and treatment side effects before and after radiation therapy. In Psycho-Oncology 17:SI-348. Retrieved from http:// www.interscience.wiley.com.

Coifman, K. G., Bonanno, G. A., Ray, R. D., \& Gross, J. J. (2007). Does repressive coping promote resilience: Affectiveautonomic response discrepancy during bereavement. Journal of Personality and Social Psychology, 92(4), 745-758.
Costa, C. R. B. S. F., \& Assis, S. G. (2006). Fatores protetivos a adolescência em conflito com a lei no contexto sócio-educativo. Psicologia e Sociedade, 18(3), 74-81.

Gimenes, M. G. G (1997). A mulher após a mastectomia: alternativas para a intervenção psicológica. In M. G. G. Gimenez (Ed.), A Mulher e o câncer (pp. 149-172). São Paulo: Editorial Psy.

Grünspun, H. (2003). Conceitos sobre resiliência. Revista Bioética, 10(1). Retrieved from http://www.portalmedico.org.br/revista/ bio10v1/seccao4.

Heim, E., Valach, L., \& Schaffner, L. (1997). Coping and psychosocial adaptation: Longitudinal effects over time and stages in breast cancer. Pscychosomatic Medicine, 59(4), 408-418.

Herman-Stahl, M., \& Petersen, A. C. (1996). The protective role of coping and social resources for depressive symptoms among young adolescents. Journal of Youth and Adolescence, 25(6), 733-753.

Herman-Stahl, M., \& Petersen, A. C. (1999). Depressive symptoms during adolescence: direct and stress-buffering effects of coping, control beliefs, and family relationships. Journal of Applied Developmental Psychology, 20(1), 45-62.

Herman-Stahl, M., Stemmler, M., \& Petersen, A. C. (1995). Approach and avoidant coping: implications for adolescents health. Journal of Youth and Adolescence, 24, 649-665.

Holahan, C. J., Moos, R. H., \& Schaefer, J. A. (1996). Coping, stress, resistance and growth: conceptualising adaptative functioning. In M. Zeidner \& N. S. Endler (Eds.), Handbook of coping: Theory, Research, Applications (pp. 24-43). New York: Wiley.

Jessor, R., Van Den Bos, J., Vanderryn, J., Costa, F. M., \& Turbin, M. S. (1995). Protective factors in adolescent problem behaviour: moderator effects and developmental change. Developmental Psychology, 6, 923-933.

Lazarus, R. S., \& Folkman, S. (1984). Stress, appraisal and coping. New York: Springer.

Lisboa, C., Koller, S. H., Ribas, F. F., Bittencourt, K., Oliveira, L., Porciúncula, L. P., \& De Marchi, R. B. (2002). Estratégias de coping de crianças vítimas e não vítimas de violência doméstica. Psicologia: Reflexão e Crítica, 15(2), 345-362.

Masten, A., \& Garmezy, N. (1985). Risk, vulnerability and protective factors in developmental psychopathology. In B. B. Lahey \& A. E. Kazdin (Eds.), Advances in clinical child psychology (pp. 1-52). New York: Plenum.

Moos, R. H. (1993). Coping Responses Inventory CRI-Adult Form Professional Manual. Flórida: Psychological Assessment Resources.

Mota, D. C.G. D., Benevides-Pereira, A. M. T., Gomes, M. L., \& Araújo, S. M. (2006). Estresse e resiliência em doença de Chagas. Aletheia, 24, 37-68.

Pesce, R.P., Assis, S.G., Avanci, J., Santos, N.C., Malaquias, J., \& Carvalhães, R. (2005). Adaptação transcultural, confiabilidade e validade da escala de resiliência. Cad. Saúde Pública, 21(2), 436-448.

Rutter, M. (1987). Psychosocial resilience and protective mechanisms. American Journal of Orthopsychiatric, 57(3), 316- 331.

Schroevers, M. J., Kraaij, V., \& Garnefski, N. (2008). The role of cognitive coping and self-regulatory process in cancer patients report of negative and positive affect. In Psycho-Oncology 17:SI-S348 Retrieved from http://www.interscience.wiley. com.. 
Sehgal, S., Hickman, S., Casden, D., Clark, K., Loscalzo, M., \& Bardwell, W. (2008). Gender differences of psychosocial distress in cancer Patients. In Psycho-Oncology 17:SI-348. Retrieved from http://www.interscience.wiley.com.

Zanini, D. S., \& Forns, M. (2005). O conceito de risco e proteção à saúde mental e sua relação com a teoria de coping. Estudos, Goiânia, 32 (1), 69-80.

Zanini, D. S., Forns, M., \& Kirchner, T. (2005). Estrategias de afrontamiento: Implicación en la salud mental de los adolescentes catalanes. Retrieved from http://www.psiquiatria. com/interpsiquis2003/9908.

Recebido em 15.07.2009 Primeira decisão editorial em 10.03.2011 Versão final em 28.03.2011 Aceito em 15.09.2011

\section{Jornadas de Formações Clínicas do Campo Lacaniano} "A Clínica do Ato

25 de Novembro de 2011

Rio de Janeiro, RJ, BR 